\title{
Construcción de ciudadanía desde la comunicación. Una mirada desde dos colectivos de jóvenes de Bogotá*
}

\author{
Delsar Roberto Gayón Tavera** \\ Sandra Ximena Gallego Galvis*** \\ José Aladier Salinas Herrera**** \\ Recibido: 2020-03-11 • Enviado a pares: 2020-03-30 \\ Aprobado por pares: 2020-05-11 • Aceptado: 2020-05-22 \\ https://doi.org/10.22395/angr.v19n37a1 1
}

\begin{abstract}
Resumen
El presente artículo muestra los resultados del proyecto de investigación Construcción de cultura política propias y ciudadanía en las prácticas comunicativas de dos colectivos de jóvenes de la localidad 18 Rafael Uribe con el propósito de aportar al debate actual sobre las relaciones entre comunicación y educación. La investigación se aborda desde el enfoque político-cultural de la comunicación que estudia las prácticas sociales y la producción de sentido, poniendo en juego la articulación de dos conceptos: ciudadanía y comunicación. Metodológicamente se basó en la etnografía autorreflexiva que permitió la descripción del contexto sociocultural y el análisis de las prácticas comunicativas de los dos colectivos en tensión con la formación escolar en asuntos relacionados con ciudadanía y paz. El principal hallazgo es que los dos colectivos tienen apuestas distintas en la producción de sentido. El Colectivo Sin Fronteras interpela en sus prácticas la formación de los procesos de enseñanza en el aula, interrogando los paradigmas de masculinidad a través de la resignificación del cuerpo, lo que permite abrir nuevas formas de vivir la ciudadanía. Por su parte, el Colectivo Somos centra sus prácticas en la construcción de una cultura de paz a través de la producción de programas radiales que asocian los contenidos a una ciudadanía que defiende los derechos de la mujer, LGBTIQ, la política, la ruptura de prejuicios y la concientización frente al trato diario en el escenario escolar.
\end{abstract}

Palabras clave: jóvenes urbanos; proceso de comunicación; educación; práctica pedagógica; cultura de paz; ciudadanía.

- Este artículo es resultado del proyecto de investigación (2018) Construcción de cultura política propias y ciudadanía en las prácticas comunicativas de dos colectivos de jóvenes de la localidad 18 Rafael Uribe Uribe adscrito al grupo de investigación Comunicación, Paz y Conflicto de la Facultad de Comunicación Social de la Universidad Santo Tomás sede Bogotá, Colombia.

* Estudiante de doctorado en Teoría Crítica en, Instituto de Estudios Críticos, Ciudad de México, México. Docente de la Facultad de Comunicación Social, Universidad Santo Tomás, Bogotá, Bogotá. Correo electrónico: delsargayon@usantotomas.edu.co. Orcid: https://orcid.org/0000-0002-4805-8565

** Doctora en Ciencias Humanas y Sociales, Universidad Nacional de Colombia, Colombia. Docente de la Facultad de Comunicación Social, Universidad Santo Tomás, Colombia. Correo electrónico: sandragallego@usantotomas. edu.co. Orcid: https://orcid.org/0000-0002-6649-4221

...* Doctor en Cultura y Educación, Universidad de Artes y Ciencias Sociales, Santiago, Chile. Docente de la Facultad de Comunicación de la Universidad Santo Tomás, Colombia. Correo electrónico: josesalinash@usantotomas. edu.co. Orcid: https://orcid.org/0000-0001-7085-9281. 


\title{
Building Citizenship from Communication. A Look from Two Young Collectives in Bogota
}

\begin{abstract}
This article displays the results of the research project Construcción de cultura politica propias y ciudadanía en las prácticas comunicativas de dos colectivos de jóvenes de la localidad 18 Rafael Uribe (Construction of own cultural policies and in the communicative practices of two young collectives from the Rafael Uribe 18th locality) to contribute to the current debate on the relationship between communication and education. The research is aided by a political and cultural approach of communication that studies the social practices and production of sense by articulating two concepts: citizenship and communication. Methodologically, this study was based in reflexive ethnography that allowed the description of the social and cultural context and the analysis of communication practices of the two collectives in tension with the school formation in matters of citizenship and peace. The main finding is that two collectives have different bets for the production of sense. In one hand, Colectivo Sin Fronteras (Without Borders Collective) interpellates with its practices the classroom formative processes, interrogating the masculinity paradigms through the resignification of the body which allows them to be open to other ways of living citizenship. In the other hand, Colectivo Somos (We are Collective) centres its practice of peace culture through the production of radio programs that associate contents with a citizenship that defends women's and LGBTO rights, policy rupture with prejudices and awareness on the day to day treatment in the school scenario.
\end{abstract}

Keywords: urban youth; communication process; education; pedagogic practise; peace culture; citizenship.

\section{Construção de cidadania com base na comunicação. Uma visão a partir dos coletivos de jovens de Bogotá}

\begin{abstract}
Resumo
Este artigo mostra os resultados do projeto de pesquisa "Construção de cultura política própria e cidadania nas práticas comunicacionais de dois coletivos de jovens da localidade 18 Rafael Uribe", com o objetivo de contribuir para o debate atual sobre as relações entre comunicação e educação. A pesquisa utiliza a abordagem político-cultural da comunicação, que estuda as práticas sociais e a produção de sentido, colocando em jogo a articulação de dois conceitos: cidadania e comunicação. Metodologicamente, está baseada na etnografia autorreflexiva que permitiu a descrição do contexto sociocultural e a análise das práticas comunicacionais dos dois coletivos em tensão com a formação escolar em assuntos relacionados com a cidadania e a paz. O principal achado está em que os dois coletivos têm apostas diferentes na produção de sentido. O Coletivo Sin Fronteras interpela em suas práticas a formação dos processos de ensino na sala de aula, interrogando os paradigmas de masculinidade por meio da ressignificação do corpo, o que permite abrir novas formas de viver a cidadania. Por sua vez, o Coletivo Somos centraliza suas práticas na construção de uma cultura de paz por meio da produção de programas de rádio que associam os conteúdos a uma cidadania que defende os direitos da mulher, os LGBTIQ, a política, a quebra de tabus e a conscientização do tratamento diário no contexto escolar.
\end{abstract}

Palavras-chave: jovens urbanos; processo de comunicação; educação; prática pedagógica; cultura de paz; cidadania. 


\section{Introducción}

Las discusiones sobre la ciudadanía presentan un panorama variopinto. En la línea clásica, Cortina (1997) propone comprender la génesis del término en cuanto a las posibilidades que tienen los individuos de pertenecer y reconocerse frente a una comunidad política. Para la autora, históricamente la ciudadanía construyó principios ideales que aún se mantienen, como la religión cívica, homo legalis, zoon politikón, convivencia, expresión y educación, principalmente. En esta misma línea, Sartori (1994) asocia la ciudadanía con la actividad de los demos dentro de los sistemas políticos que contribuyen a la construcción de democracia. Por una parte, debe entenderse la ciudadanía como una entidad jurídica definida por el Estado y como un estatus concedido a los individuos, es decir, un derecho otorgado. Por otra parte, en la línea de Cortina (2001), se propone construir el concepto partiendo de considerarla como una entidad orgánica de la sociedad, en la cual coexisten diferentes grupos humanos en distintas actividades.

Lechner $(1990,2000)$ afirma que la transición de una ciudadanía clásica a las denominadas nuevas ciudadanías generó tres rupturas: la primera se refiere a nuevas formas de ejercicio ciudadano y de relaciones entre el individuo y el Estado; la segunda se circunscribe al ámbito de la territorialidad en nuevos espacios sociales y urbanos; la tercera hace referencia a los intereses de diverso orden por los que se convocan y movilizan hoy los ciudadanos. En palabras de Salazar (2013), se vive la construcción de un nuevo sujeto político en la sociedad contemporánea encarnado en los jóvenes y las mujeres. Además, valida la concepción de ciudadanía de T.H. Marshall, en cuanto sea activa en la vida social a través de prácticas ciudadanas que supongan el ejercicio de los derechos civiles, políticos y sociales en la experiencia de vida (Buenrostro, 2012).

En esta misma óptica, Dietz (2012) y Quesada (2008) resaltan las transformaciones culturales contemporáneas que han posibilitado la organización y expresión de colectivos de distinta naturaleza: estudiantiles, urbanos, feministas, ecologistas y pacifistas con características que incluyen cierta flexibilidad en las jerarquías, rechazo al autoritarismo, autonomía frente al Estado, los partidos políticos y sin una ideología específica. Estos colectivos han determinado otras modalidades en el ejercicio de la ciudadanía porque, además de los contenidos de las demandas dirigidas al Estado, generaron formas inéditas de expresión y organización para promoverlas. Para Rueda (2008), la emergencia de estas formas de expresión son consecuencia de procesos tecnosociales que transforman la sensibilidad, la ritualidad, las relaciones sociales, las narrativas culturales y las instituciones políticas.

En este contexto mediático y tecnológico de la ciudadanía se han introducido distinciones entre lo público y lo privado. Para Huergo (2013), lo público es un espacio fluido y polimorfo ligado a los medios, que garantiza la opinión pública; así, lo 
público se constituye en espacio mediático. Con respecto a las articulaciones entre la ciudadanía y lo tecnosocial, Martínez y Burgos (2014) hablan de ciudadanías comunicativas para referirse a la pluralidad cultural, los procesos de expresión, información, comunicación y recepción, es decir, un asunto de medios, mediaciones y prácticas cotidianas para garantizar su ejercicio de construcción real y permanente. Tamayo y Navarro (2018) comparten esta visión y agregan nuevos elementos como la emergencia de agencias de ciudadanías comunicativas que alientan la participación más activa de los ciudadanos en la construcción de sus regímenes sociocomunicativos, la construcción de una esfera pública diversa e incluyente y la expresión pública de sus demandas y puntos de vista. Además, permite el reconocimiento público de sus relatos, pensamientos e intereses en el espacio público.

En las últimas décadas se abrió una nueva vertiente de estudios que relaciona cultura política y educación ciudadana. En Colombia, estas investigaciones (Herrera et al., 2005) se dividen en al menos dos vertientes: el liberalismo, que reconoce en el Estado el referente para la elaboración de los fundamentos político-culturales desde los que debería educarse a los ciudadanos, y la óptica conservadora, con la Iglesia como protagonista, que piensa los comportamientos cívicos basados en valores de tipo moral y religioso.

Sánchez (2017) toma como punto de partida la importancia del concepto de transformación activa. Este concepto se entiende como el cambio de rol que realizan las personas o colectivos: pasan de ser sujetos o grupos pasivos o inactivos socialmente a ser propositivos y ejecutores de acciones que beneficien el desarrollo de sus entornos.

El presente artículo aporta a este debate a partir la siguiente pregunta: ¿Cómo las prácticas comunicativas de los colectivos Sin Fronteras y Somos construyen ciudadanía y cultura política propaz, en tensión con la educación escolarizada de dos instituciones distritales de Bogotá?

Este interrogante se respondió tomando como objetivo general evidenciar las prácticas comunicativas de los dos colectivos y sus procesos de construcción de ciudadanía y cultura política propaz, en tensión con la educación escolarizada impartida en los colegios distritales Clemencia Holguín de Urdaneta y Liceo Femenino Mercedes Nariño. Se plantearon como objetivos específicos: I) reconocer el contexto sociocultural mediante el cual los colectivos construyen sus referentes sobre paz y ciudadanía; ii) analizar en las prácticas comunicativas de los colectivos las formas de expresión propaz y de ciudadanía en el espacio público urbano y en redes digitales; iii) identificar la existencia de tensiones entre los modelos tradicionales de educación democrática y las construcciones de paz y ciudadanía que emergen de sus prácticas comunicativas. 


\section{Metodología}

La investigación inició con la exploración de dos fuentes primarias para la consecución de los colectivos en actividad en la localidad Rafael Uribe. La primera fuente fue una base de datos proporcionada por el Instituto Distrital para la Protección de la Niñez y la Juventud (Idipron, s.f.). En esta base se referenciaron un total de cuarenta y un colectivos. Sin embargo, al tratar de establecer contacto se evidenció que la información estaba desactualizada y, por lo tanto, se invalidó el uso de esta fuente.

La segunda fuente, la Corporación Vínculos, informó sobre la existencia del Colectivo Sin Fronteras integrado por estudiantes del Colegio Clemencia Holguín de Urdaneta. Y como tercera fuente se contactó al director del Colectivo Somos integrado por estudiantes del Liceo Femenino Mercedes Nariño.

Se procedió a verificar, mediante trabajos con grupos focales, que los colectivos cumplían con las siguientes condiciones: compartir objetivos comunes, tener una identidad, identificar mínimo un opositor y promover modelos sociales alternativos que expresen nuevas posibilidades de participación y organización ciudadana. Este ejercicio permitió elegir sujetos de estudio al Colectivo Somos y el Colectivo Sin Fronteras, ya que ambos tienen como una de sus preocupaciones centrales el debate y los usos estratégicos del lenguaje, en función de la construcción de la cultura de paz.

Luego de la fase exploratoria, se aplicó el enfoque político cultural de la comunicación con Torrico (2004) y Reguillo (1991) para estudiar las prácticas comunicativas. Para la metodología de la autorreflexividad con los actores sociales, se trabajó la propuesta de la etnografía y reflexividad de Guber (2006) y el enfoque de ciudadanías comunicativas de Martínez y Burgos (2014) para identificar las percepciones de ciudadanía y paz que tienen los dos colectivos. Como resultado de esta elección metodológica, se implementaron las siguientes fases:

Primero se realizó un reconocimiento del contexto sociocultural. Se consideraron las variables "breve historia de la localidad", "características poblacionales, censo de colegios de educación media en la localidad Rafael Uribe". Las fuentes primarias utilizadas fueron documentos de la Alcaldía Mayor de Bogotá (2017), Secretaría de Salud de Bogotá (2011), Alcaldía Local Rafael Uribe (2018), Secretaría de Educación (2016) y documentos institucionales del Liceo Femenino Mercedes Nariño y del colegio Clemencia Holguín de Urdaneta. Por razones de espacio, en este artículo no se contemplan los hallazgos sobre el contexto histórico, demográfico y de oferta educativa de la localidad.

En segundo lugar, se hizo una cartografía de las prácticas comunicativas. Como se mencionó anteriormente, se siguió la propuesta de Reguillo (1991) sobre los usos 
sociales de la comunicación que contemplan técnicas para investigar la producción colectiva de sentido; la interacción entre actores e investigadores; la observación de la producción; los productos comunicativos y la circulación social de esta producción.

En la fase de recolección de información se trabajó con un total de 30 miembros de los dos colectivos. El abordaje del tema de la producción de la comunicación y las ciudadanías comunicativas se llevó a cabo mediante dieciocho talleres con los siguientes objetivos: reconstruir la historia de los colectivos mediante la elaboración de líneas del tiempo, reconocer el uso social de la comunicación que hacen los dos colectivos y establecer las percepciones de ciudadanía y paz.

También se realizaron seis entrevistas semiestructuradas a docentes de cada colegio para profundizar la información obtenida y a cuatro integrantes de los colectivos con el objetivo de evaluar de mejor manera sus motivaciones sobre la pertenencia a los mismos.

El paso final fue la sistematización de la información recolectada en los talleres y las entrevistas a través de una matriz de análisis cualitativo mediante las siguientes categorías:

- Expresividad-producto de la comunicación: se entiende la expresividad con Martínez y Burgos (2014) como posibilidad de exteriorizar un discurso a través de diferentes medios y lenguajes en búsqueda de un interlocutor. Y con Reguillo (1991) se entiende como un producto de la comunicación, en cuanto a formas de producción materializada en piezas comunicativas como representación y sentido de la realidad, es decir, que poseen un valor social.

- Informatividad-circulación: es la puesta en circulación social de los productos comunicativos a través de la ecología mediática que esté al alcance de los actores sociales (Martínez y Burgos, 2014, Reguillo, 1991).

- Comunicabilidad: es la relación comunicativa en cuanto a confrontación, intercambio, negociación, resistencia o conciliación entre actores sociales legitimados como interlocutores de este encuentro (Martínez y Burgos, 2014).

- Receptividad: entendida como la capacidad crítica y activa de los receptores de los mensajes (Martínez y Burgos, 2014; Reguillo, 1991).

\section{Resultados}

\section{Colectivo Sin Fronteras}

En la reconstrucción de la historia del Colectivo Sin Fronteras se realizaron, con la orientación de los investigadores, tres talleres de autorreflexividad entre sus miembros. 
Se utilizó la técnica de líneas del tiempo con dos objetivos: conocer el surgimiento y transformación del colectivo, y reconstruir la historia de su activismo en línea o fuera de línea.

En cuanto a la configuración del colectivo, la primera línea del tiempo estableció que este ha sufrido cuatro transformaciones desde su fundación en 2006. En su etapa inicial se denominó Colectivo Fronteras Tras Fronteras, Derechos sin Fronteras. En un segundo momento, se autodenominó Proyecto Sin Fronteras. Estuvo conformado por estudiantes de sexto a once grado que realizaron actividades durante cinco años. En un tercer momento, se crea el Colectivo Sin Fronteras conformado por los estudiantes y egresados del colegio Clemencia Holguín de Urdaneta en alianza con el Colectivo Nuevas Masculinidades que hace presencia en varias regiones de Colombia.

En lo referido a los hechos liderados por el colectivo, se reconstruyeron cuarenta y nueve actividades entre los años 2013-2018. Las principales fueron: trece marchas, quince salidas de campo, seis actividades oficiales en el colegio, tres plantones, dos campañas, dos campamentos, dos festivales, cuatro participaciones en eventos, una performance y una velatón. Se destacan como fechas memorables la participación en actividades oficiales en el colegio, como el día de la mujer y el día de la tierra. De las intervenciones del colectivo en espacios públicos, se recuerdan la participación en la marcha de las putas, las marchas contra el feminicidio, las mingas por la niñez, el Foro Educativo Institucional Local y Distrital, la velatón por los líderes sociales y la marcha de las faldas. Con respecto a las relaciones entre el activismo online-offline, se encontró una concordancia porque su muro de Facebook es un lugar de la memoria del activismo en el espacio público en temas de sexualidad, género y nuevas masculinidades. Se postean, antes y después de las apariciones en el espacio público, expresiones como: "Símbolo de afecto con un verdadero significado de hermandad, apoyo y amor entre las nuevas masculinidades y las nuevas feminidades" (Colectivo Sin Fronteras, 2014).

Por otra parte, los resultados de los usos sociales de la comunicación se obtuvieron mediante la realización de cuatro talleres. Se estableció que en el Colectivo Familia Sin Fronteras no existen relaciones jerárquicas y, en el desarrollo de las actividades, los roles de funcionamiento son intercambiables. El colectivo ha mantenido, a lo largo de los años, tres ejes temáticos de trabajo: convivencia, sexualidad y cuerpo. El compromiso de cada participante es convertirse en multiplicador, dentro y fuera de la institución, de las prácticas ciudadanas propaz. Los resultados de estas prácticas se evidencian en la disminución de embarazos en adolescentes, reducción de la violencia de género y mayor integración, sin distinción de género, entre los estudiantes del colegio en actividades lúdicas como recreos, festivales y celebraciones (Colectivo Familia sin Fronteras, 2018). 
Otro aspecto de los usos sociales es la concepción de la comunicación como una práctica social, no siempre mediática, expresada en el desarrollo de una metodología autónoma por parte de los miembros del colectivo, cuyo principio fundamental es su carácter vivencial. De acuerdo con la información obtenida en los talleres, los registros sonoros, audiovisuales y el material escrito, la metodología referida tiene las siguientes fases:

Se parte de hacer práctica la teoría. Este ejercicio se basa en llegar a los jóvenes de una forma distinta, motivándolos y mostrándoles que no solo se aprende por medio de la teoría, sino con ejercicios de activación corporal que permitan abordar el tema de los derechos sexuales y reproductivos. Se complementa con la dinámica de rompehielos para abordar el tema de los derechos humanos para lograr una mejor comprensión de los temas y conseguir un mayor rendimiento con el grupo trabajado (Colectivo Familia Sin Fronteras, 2018).

Otra fase que consiste en vivenciarse a sí mismo desde una perspectiva relacional de género (nuevas masculinidades y feminidades). Esta dinámica favorece experiencias analíticas y vivenciales de los integrantes del colectivo que les permita reconciliarse como seres humanos que aman, quieren igualdad y luchan por la convivencia y la paz: "Encontrémonos con nuestro niño interno, seamos seres humanos" (Colectivo Familia Sin Fronteras, 2018, p. 9). En esta fase también se utilizan la animación y la pintura corporal como convivencia mágica.

La penúltima fase es la cohesión y liderazgo que busca la transformación positiva de los conflictos, tanto al interior como fuera de la institución educativa, en escenarios como el barrio, la familia y los grupos de amigos. La metodología culmina con el ritual de cierre que busca que los participantes elaboren una biografía social y sexual de hombres y mujeres bajo el modelo patriarcal.

En cuanto a la producción de la comunicación mediática, los miembros del colectivo reconocieron que no han sido tan conscientes de la importancia de la elaboración de piezas comunicativas como parte de su activismo político. En los archivos se identificaron dos piezas comunicativas relevantes que refieren a la identidad del colectivo (figuras 1 y 2).

En la revisión se encontraron otras piezas que no han sido sistematizadas por ninguna generación del colectivo, tales como folletos, fotografías documentales, memorias audiovisuales de aparición en canales de televisión y performances.

Las dos últimas jornadas de trabajo se orientaron a establecer las percepciones sobre el tema de ciudadanía y paz. En la primera jornada, los miembros del colectivo pusieron sobre la mesa el tema de autorreflexión sobre los imaginarios que se tienen sobre ellos al interior del Colegio Clemencia Holguín de Urdaneta, como personas gay 
y lesbianas. En esta jornada expresaron la percepción que tienen del tema de ciuda danía y paz propuesto en el proyecto educativo institucional del colegio. La figura 3 sintetiza esta percepción.

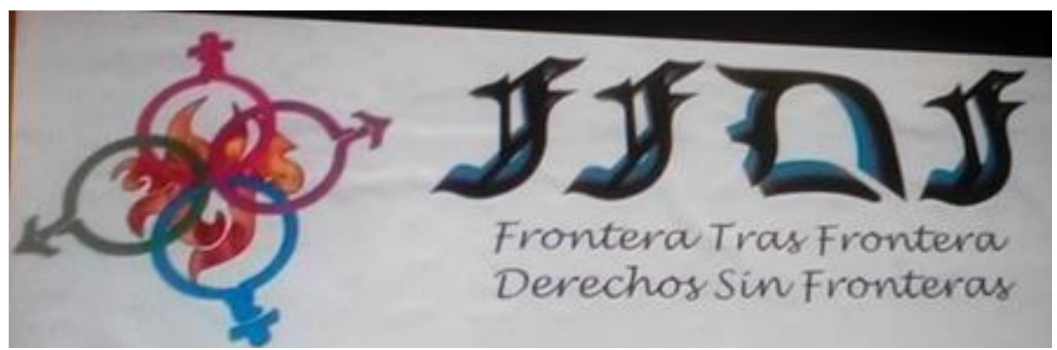

Figura 1. Logo colectivo Frontera tras Frontera Derechos sin Frontera

Fuente: Colectivo Sin Fronteras, archivo personal, 2018.

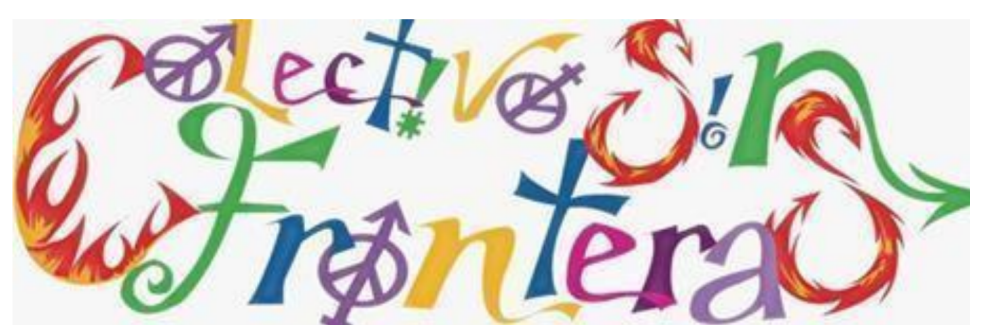

Figura 2. Logo Colectivo Sin Fronteras

Fuente: Colectivo Sin Fronteras, archivo personal, 2018.

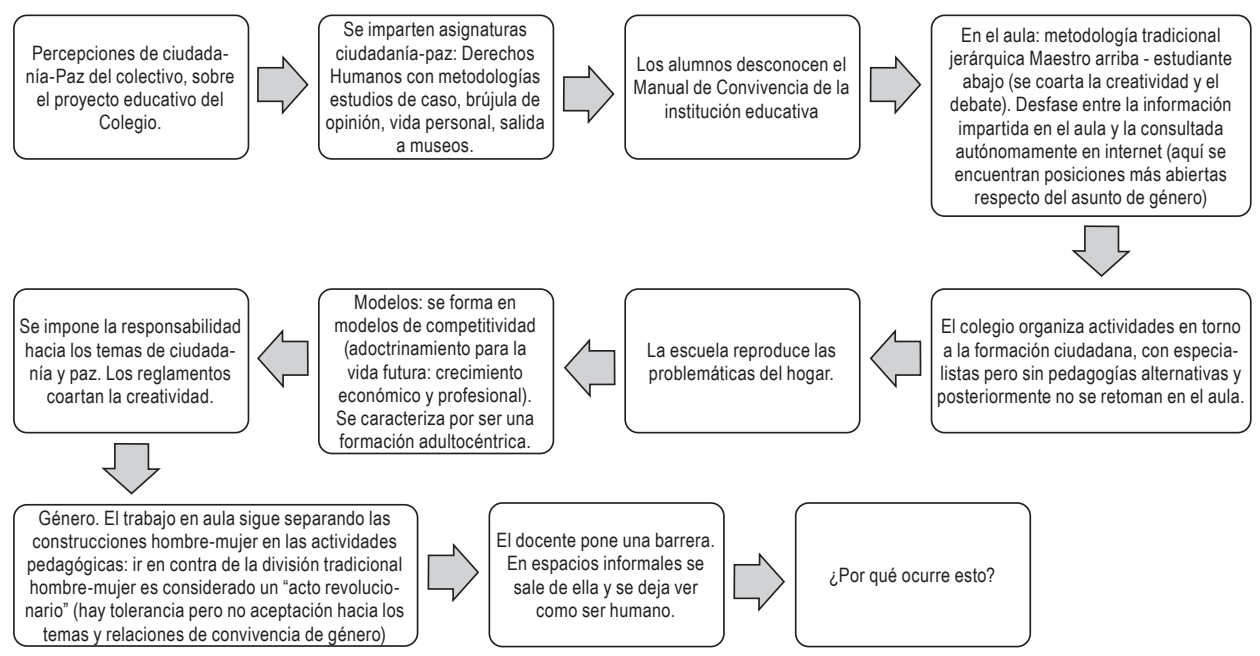

Figura 3. Percepciones de ciudadanía y paz desde el proyecto educativo del Colegio Fuente: elaboración propia. 
En la última jornada se expusieron las percepciones de ciudadanía y paz construidas al interior del colectivo. Las figuras 4 y 5 dan cuenta de ello.

Percepción de ciudadanía y paz desde colectivo

Consideran las actividades del colectivo como pedagogía alternativa: son vivenciales (primero sentir, luego pensar: la corporalidad hace sentir al otro, encontrarse como sujetos, el cuerpo es un medio para romper paradigmas patriarcales y construye convivencia).

Se reconocen tres construcciones del cuerpo: el cuerpo visibilizado con fines comerciales, el cuerpo como potenciador de relaciones sociales de convivencia, el cuerpo como modo de expresión.

A partir del respeto por las opiniones, se promueve la libre expresión, permite formación de pensamiento colectivo y de convivencia.

El colectivo procura otro modelo de estudiante en la institución que aún no es reconocido por parte de docentes y administrativos. Permite un mayor reconocimiento de la institución educativa.

Se reconoce el ejercicio de la ciudadanía sobre todo como el derecho a estar y expresarse libremente en el espacio público.

Cercanía entre las actividades del colectivo y los contenidos de las nuevas tecnologías como internet.

Figura 4. Percepción de ciudadanía y paz desde el colectivo.

Fuente: elaboración propia.
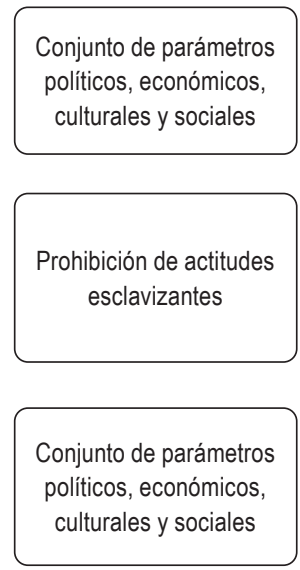
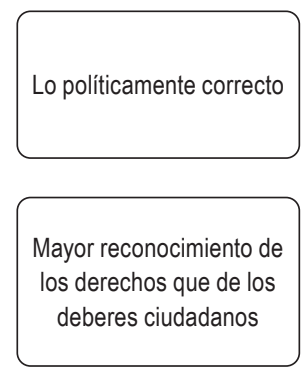

El colectivo es un espacio de paz: escucha, respeto por la opinión, sana convivencia y aceptación del a diferencia sexual.
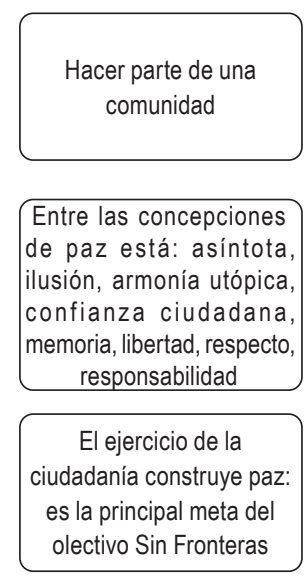
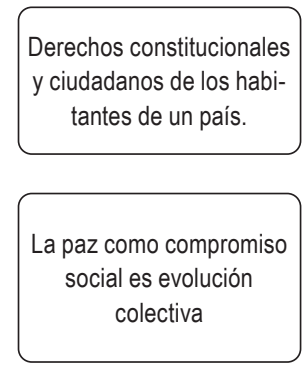

Derechos constitucionales y ciudadanos de los habitantes de un país.

Figura 5. Concepciones de ciudadanía y paz que tienen los distintos integrantes del colectivo Fuente: elaboración propia.

\section{Colectivo Somos}

La reconstrucción de la historia del Colectivo Somos se trabajó en tres talleres, utilizando la misma técnica de la línea del tiempo. El colectivo inicia en el 2016 en el marco de la celebración de los cien años del Liceo Femenino Mercedes Nariño, en la jornada de la tarde. En este contexto, algunos docentes se plantearon la pregunta, ¿qué significa educar niñas y jovencitas del presente siglo? Bajo este interrogante, se convocan a las estudiantes que deseen participar en un grupo que aborde el tema de 
la educación de la mujer y genere una expresión crítica de su pensamiento a través de un lenguaje estético y mediático. Inicialmente, hicieron parte del colectivo doce alumnas de noveno grado y, durante los años 2017 y 2018, se amplió a los niveles séptimo, octavo y once con una participación alrededor de sesenta estudiantes de diversos cursos y de las jornadas de la mañana y la tarde. En este contexto, el colectivo eligió, como escenario de expresión, la emisora escolar del colegio. En los meses siguientes, con el apoyo de Comunicarte y de estudiantes del programa Comunicación Social de la Universidad Santo Tomás de Bogotá, se capacitó a las integrantes en producción radial. Este proceso se sintetiza en la siguiente línea del tiempo (figura 6):

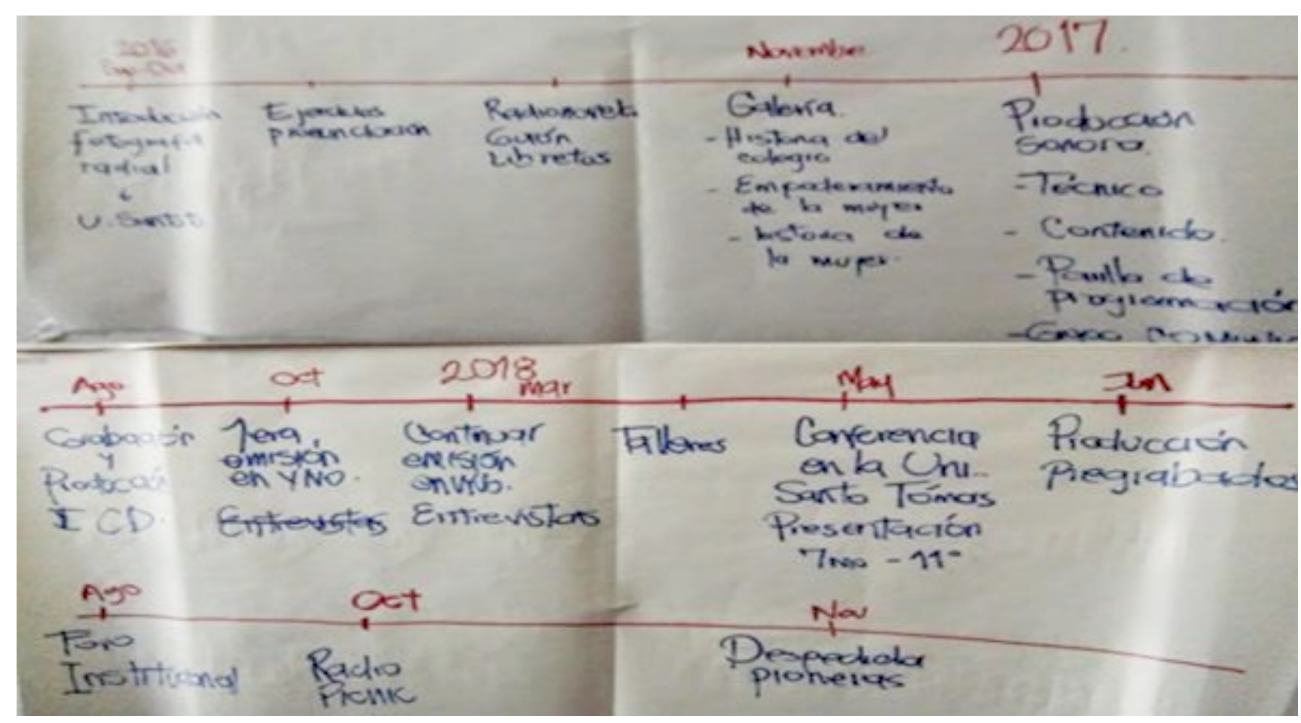

Figura 6. Línea del tiempo

Fuente: Colectivo Somos (2018).

Los resultados sobre los usos sociales de la comunicación se obtuvieron mediante la realización de cuatro talleres. En el primero, se evidencia la comunicación como una práctica social que convoca la discusión alrededor de temas como la ciudadanía, la participación y el género. La denominación somos, que da nombre al colectivo, representa el empoderamiento de las participantes como ciudadanas con posibilidad de construir sentidos sobre la identidad y formación de la mujer en contextos comunicativos y culturales. Muestra de ello son los objetivos de trabajo y la definición de una identidad gráfica (figura 7):

- Reconocer a las niñas y jóvenes liceístas desde su propia sensibilidad.

- Abrir un espacio de comunicación y expresión del pensamiento juvenil, a partir de los lenguajes mediáticos y estéticos. 


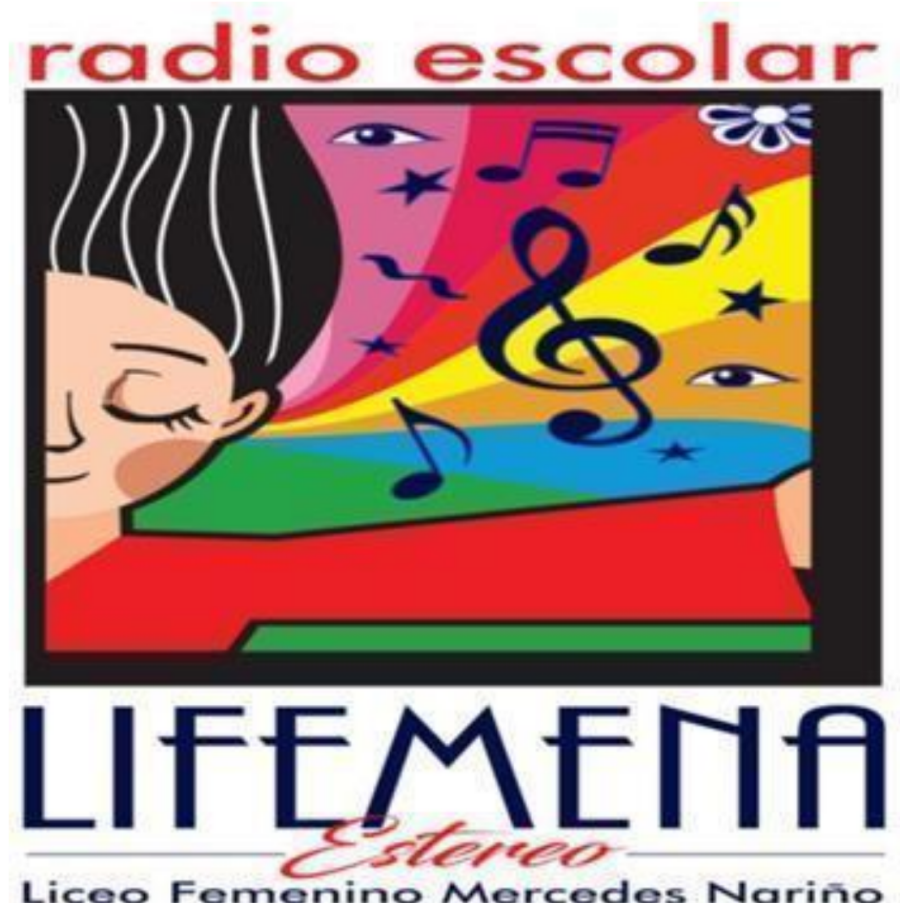

Figura 7. Poster de la radio escolar Lifemena

Fuente. Colectivo Somos, archivo personal, 2018.

Otro taller estuvo dedicado a las condiciones de producción comunicativa del colectivo. Resultado del trabajo de auto reflexividad, se identificaron los siguientes temas: LGBTIO, historias de vida, reconocimiento de días importantes en la historia, concientización ambiental, debates sobre la libertad de culto y la religión, luchas sociales, violencia de género, acoso escolar, maltrato animal, abuso sexual, música protesta, feminismo, política, estereotipos, estigmas sociales, comunidad lifemena y relación de las liceístas con su entorno.

Las estudiantes llegaron a la conclusión de que los temas que mejor definían su objetivo como colectivo era la relación entre la mujer y la política. Las participantes argumentaron que, al ser un colegio femenino, se hace indispensable pensar en el papel que ocupa la mujer en la sociedad actual. Ven la necesidad de tener una alta incidencia política para modificar los aspectos de inequidad existentes en temas de género.

Sobre la producción radial, hubo consenso en el análisis de tres aspectos: emisiones en vivo, producciones grabadas y radio picnic. En cuanto a las emisiones en vivo, se destacó que es un proceso que les permitió entender que la emisora no es solo música, sino también algo que permite llevar mensajes para ayudar y ser del interés común (Colectivo Somos, 2018). En relación con las producciones grabadas, se des- 
tacó el aprendizaje en medio del proceso y la importancia de seguir trabajando sobre la reflexión frente al papel de la mujer en la sociedad y, sobre todo, la oportunidad de dar apertura a temáticas que antes no habían sido tratadas tan abiertamente en la Institución Educativa, como lo referente a la comunidad LGBTIO.

En cuanto al radio picnic, las integrantes rescataron las habilidades adquiridas para realizar sus presentaciones en vivo, la experiencia adquirida en la planeación de los programas y el trato directo con invitados externos. Se resaltó la oportunidad de abrir espacios de debate y reflexión en temas de interés para todas las liceístas referentes al género, el cuidado animal y medioambiental, el feminismo, el cutting y la violencia sexual. En la figura 8 se aprecia la autorreflexión llevada a cabo por las estudiantes:

\section{Radio Picnic}

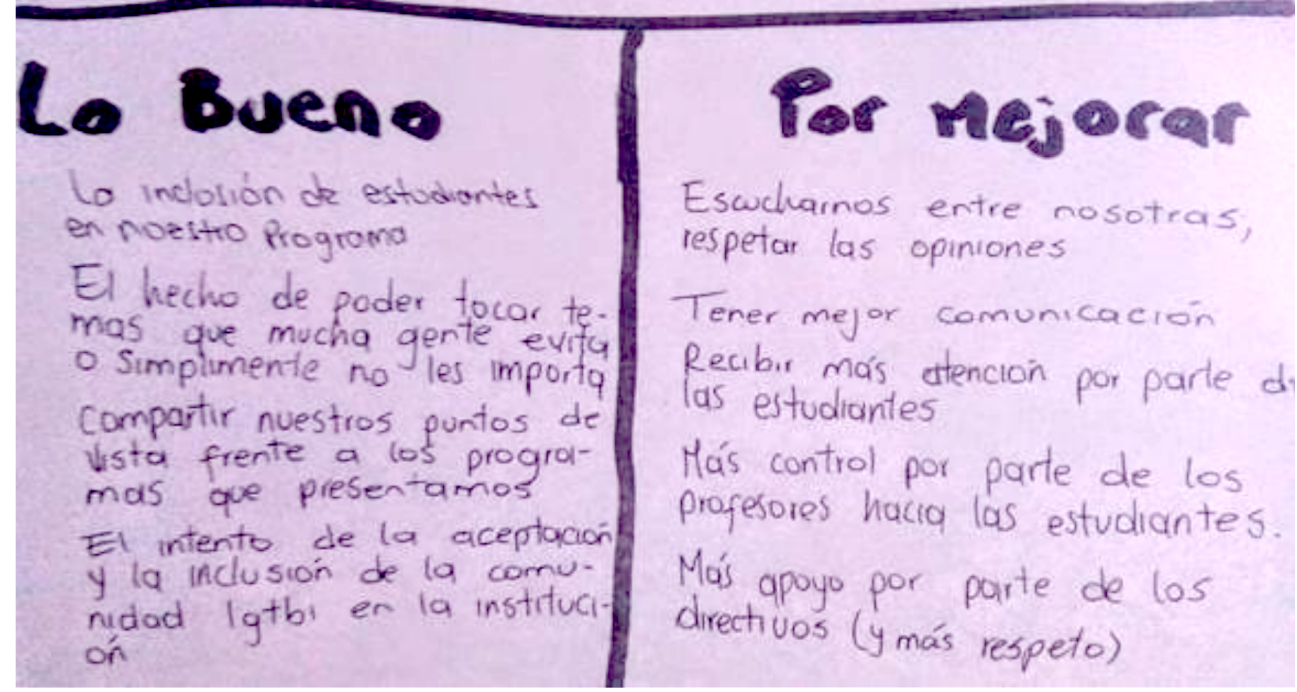

Figura 8. Evaluación actividad Radio Picnic

Fuente: Colectivo Somos (2018).

En los dos últimos talleres, se llevó a cabo una autorreflexión sobre ciudadanía y paz. Se destacan algunas temáticas en cuanto a la definición de la categoría de ciudadanía en su contexto actual: derechos de la mujer, LGBTIO, violencia, política, ruptura de prejuicios y concientización. En cuanto a la categoría de la paz, se señaló que ésta empieza por las relaciones de convivencia de las participantes entre sí, como desde su trato diario con otras personas. En cuanto a la comunicación, se señaló que es un proceso para la escucha de diversas voces, el reconocimiento del otro y facilitar la comprensión de la alteridad. 


\section{Discusión y conclusiones}

Siguiendo la propuesta de ciudadanías comunicativas de Martínez y Burgos (2014) y los usos sociales de la comunicación de Reguillo (1991), se puede concluir que los dos colectivos estudiados despliegan ciudadanías comunicativas en cuatro niveles:

1. Expresividad- producto de la comunicación. En esta dimensión los dos colectivos, a través distintas formas de expresión, ponen en común sus preocupaciones en torno al tema de la ciudadanía y la paz. El Colectivo Sin Fronteras ha realizado un proceso de autorreflexividad sobre la construcción de nuevas masculinidades y la resignificación del cuerpo como posibilidad de romper paradigmas patriarcales, ser ciudadano y cómo estas prácticas aportan a la convivencia escolar, familiar, local y nacional. En el mismo ejercicio, el Colectivo Somos evidencia una preocupación por el trabajo de la construcción de una cultura de paz, pues las actividades alrededor de la emisora escolar se caracterizan por asumir el reconocimiento de los otros-otras desde el carácter feminista del colectivo. En este sentido, las actividades buscan valorar las distintas opiniones, expresiones y reconocimiento de todas las estudiantes.

2. Informatividad-circulación. Las prácticas comunicativas de los colectivos muestran intereses distintos en cuanto a su capacidad de acción informativa y de posturas críticas como receptores frente a los temas de ciudadanía y paz. Así, el Colectivo Familia Sin Fronteras no ha tenido en sus temas de trabajo la reflexión sobre la comunicación, sino que ve a la escuela como un escenario de reproducción de la tradición cultural del Estado, la familia y la Iglesia, con prácticas adultocéntricas y jerárquicas. En este sentido, este colectivo ve a la ciudadanía como un derecho constitucional, pero empaquetada en un conjunto de normas preestablecidas. Asimismo, ve la paz como un estado utópico, pero alcanzable mediante el ejercicio responsable de la ciudadanía.

El Colectivo Somos parte de reconocer el lenguaje como el más importante medio de conocimiento que tiene el ser humano y ven la comunicación como un proceso mediante el cual pueden dar a conocer sus propias voces y las voces de las demás. Las estudiantes ven a la ciudadanía potencialmente favorable en su contexto actual y la asocian con los derechos de la mujer. En cuanto a la paz, señalaron que esta se construye cotidianamente y la connotan como un estado de convivencia a nivel general.

3. Comunicabilidad. El Colectivo Familia Sin Fronteras no tiene una producción planeada y constante en cuanto a productos comunicativos como apoyo a sus formas de acción. Sin embargo, en el trabajo de campo se concluyó que su comunicabilidad está centrada en la aparición en los espacios de socialización del colegio, en el espacio público urbano y en la participación de actividades con otros 
colectivos, ONG como la Corporación Vínculos y en algunos eventos académicos. En cambio, el Colectivo Somos centra sus prácticas en la producción radiofónica y en la construcción de relatos que favorecen la convivencia pacífica a través de la ética, los valores, la moral y las diferentes responsabilidades ciudadanas.

4. Receptividad. En cuanto a producción de sentido activo y crítico en el rol de receptor de mensajes tanto mediáticos como no mediáticos, el Colectivo Sin Fronteras se muestra crítico frente a la formación ciudadana y pacífica impartida desde las instituciones (familia, escuela, estado, medios masivos). En este sentido, trabaja por la construcción de una nueva lógica de la juventud que piense y sienta la vida sin menos prejuicios de autoridad, género. Así, lleva a la esfera educativa y pública la construcción de nuevas masculinidades que ellos mismos llaman liberadoras y libertarias (Colectivo Familia Sin Fronteras, 2018).

La receptividad crítica del Colectivo Somos se enfoca en el tema de la mujer y la política. Pasan de ser una audiencia pasiva de las narrativas institucionales para convertirse en un colectivo, a través de la radio escolar, para que las adolescentes entiendan la importancia de reivindicar y hacer respetar sus derechos como mujeres. Ven la necesidad, a través de la comunicación, de tener una alta incidencia política para contribuir a modificar la inequidad de género.

Para finalizar, se puede evidenciar en el trabajo con los colectivos estudiados que no necesariamente los escenarios de educación escolar y los escenarios no escolarizados como los colectivos Sin Fronteras y Somos, se excluyen mutuamente en las prácticas de educación ciudadana. En este hallazgo se coincide con las investigaciones de Moreno (2018) y Narváez (2004) en tanto se necesitan mayores investigaciones para resignificar las relaciones entre comunicación, cultura escolar y educación no formal. Esto se debe a que, como lo plantea Valderrama et al. (2003), la compleja densidad simbólica de la vida cotidiana interpela a la escuela para que incorpore, en sus lógicas institucionales y pedagógicas, las manifestaciones de la cultura contemporánea.

La comunicación resulta un eje articulador y dinamizador para acercar estos dos procesos formativos. En este sentido, abre espacios al interior de las instituciones educativas para construir experiencias y debates sobre la función política de la comunicación verbal y no verbal: la desconstrucción y construcción de discursos alternativos sobre el cuerpo que conllevan a cambiar las relaciones de convivencia en la vida escolar y cotidiana. Otros escenarios de formación de ciudadanías críticas se generan en los medios escolares, performances o en la participación en discusiones sobre estos temas en el espacio público urbano y en programas de opinión en los medios de comunicación masiva. Esto coincide con Cubides y Forero (2018) al plantear la novedad y potencia ética de las prácticas y saberes de redes de colectivos sociales, las modalidades de pensamiento que despliegan y el tipo de reflexividad que efectúan. 


\section{Referencias}

Alcaldía Local de Rafael Uribe. (2018, 15 de noviembre). Portal web. Consultado el 22 de febrero de 2019. http://www.rafaeluribe.gov.co/

Alcaldía Mayor de Bogotá. (2017). Caracterización general de escenarios de riesgo. Localidad Rafael Uribe. Consejo de gestión de riesgo y cambio climático.

Alcaldía Mayor de Bogotá y Secretaría de Salud de Bogotá. (2011). Localidad Rafael Uribe (2009-2010). Diagnóstico local con participación social. Alcaldía Mayor de Bogotá. http://www.saludcapital.gov. co/sitios/VigilanciaSaludPublica/Diagnosticos\%20Locales/18-RAFAEL\%20URIBE\%20URIBE.pdf

Buenrostro Sanchez, I. (2012) La ciudadanía de T.H. Marshall. Apuntes sobre un concepto sociológico olvidado. En S. Gallego y E. Díaz (coords.), X Premio de Ensayo Breve "Fermín Caballero" (pp. 59-84). Asociación Castellano Manchenga de Sociología.

Colectivo Familia Sin Fronteras. (2018). Presentación del colectivo. Colegio Clemencia Holguín de Urdaneta.

Colectivo Sin Fronteras. (2014). Símbolo de afecto con un verdadero significado de hermandad, apoyo y amor entre las nuevas masculinidades y las nuevas feminidades. [Publicación de estado]. Facebook. https://web.facebook.com/ColectiivoFFDF/photos/a.262673243913936/264621230385804/ ?type $=3 \varepsilon_{\text {theaterE_rdc }}=1 \varepsilon_{-}$rdr

Colectivo Somos. (2018). Taller: la comunicación, prácticas y productos comunicativos. Liceo Femenino Mercedes Nariño.

Cortina, A. (2001). Ciudadanos del mundo. Hacia una teoría de la ciudadanía. Alianza

Cubides, H. y Forero, G. (2018). Experiencia, saber-hacer y la construcción práctica de redes de colectivos sociales. Nómadas (49), 69-85.

Dietz, G. (2012). Multiculturalismo, interculturalidad y diversidad en educación.: Fondo de cultura económica.

Guber, R. (2006). La etnografía: método, campo y reflexividad. Norma.

Herrera, M., Pinilla, A., Díaz, C. e Infante, R. (2005). La construcción de cultura política en Colombia. Proyectos hegemónicos y resistencias culturales. Universidad Pedagógica Nacional.

Huergo, J. (1998). Las alfabetizaciones posmodernas, las pugnas culturales y los nuevos significados de la ciudadanía. Nómadas (9), 49-60.

Instituto Distrital para la Protección de la Niñez y la Juventud, Idipron(s.f.). Directorio de unidades de protección integral. Alcaldía Mayor.

Lechner, N. (1990). Los patios interiores de la democracia. Subjetividad y política. Fondo de Cultura Económica.

Lechner, N. (2000). Nuevas ciudadanías. Revista de Estudios Sociales, (5), 25-31.

Moreno, E. (2018). Aproximación al estado del arte sobre comunicación y educación en Colombia. Anagramas. Rumbos y sentidos de la comunicación, 17(33), 251-274. 
Martínez, R. y Burgos, P. (2014). Ciudadanías comunicativas y construcción de paz: la Agenda de Paz de Nariño. Signo y Pensamiento, 33(65), 32-46.

Narváez, A. (2004). Cultura mediática y educación formal: un punto de vista comunicacional. Revista Colombiana de Educación (46), 80-115.

Quesada, F. (2008). Ciudad y ciudadanía Senderos contemporáneos de la filosofía política. Trotta.

Reguillo, R. (1991). En la calle otra vez. Las bandas: identidad urbana y usos de la comunicación. Iteso.

Rueda, R. (2008). Ciberculturas: metáforas, prácticas sociales y colectivos en red. Nómadas, (28), $8-20$.

Salazar, R. (2013). La construcción del nuevo sujeto político en América Latina. Estrategia para buscar la emancipación de lo popular en el siglo XXI. Espiral, XX(57), 155-173.

Sánchez, J. R. (2017). La comunicación participativa como herramienta generadora de ciudadanía activa en la niñez [tesis de doctorado, Universidad Complutense de Madrid]. Repositorio Universidad Complutense.

Sartori, G (1994). ¿Qué es la democracia? Anagrama.

Secretaría de Educación de Bogotá (2016). Plan Sectorial de Educación. Alcaldía Mayor de Bogotá. https://www.educacionbogota.edu.co/portal_institucional/sites/default/files/inline-files/ Plan_sectorial_2016-2020-.pdf

Tamayo, C. y Navarro, D. (2018). Cuando la razón no lo explica todo: acciones de ciudadanías comunicativas en contexto de conflicto armado o violencia desde una mirada transnacional. En Palabra Clave, 21(4), 1107-1136.

Torrico, E. (2004). Abordajes y períodos de la teoría de la comunicación. Norma.

Valderrama, H. Rojas, C., Castellanos, S. y Melo, A. (2003). Comunicación, educación y ciudadanía. Discursos de actores escolares. Nómadas (18), 239-241. 